\title{
Dr. Erol Pohlreich* \\ Besonderheiten im Recht der Strafvollstreckung und des Strafvollzugs bei ausländischen Straftätern
}

\section{Einleitung}

Im Jahr 2013 befanden sich in Deutschland 56.641 Strafgefangene und Sicherungsverwahrte in $\mathrm{Haft}^{1}$, davon waren 13.248 - also fast jeder Vierte - staatenlos oder nichtdeutscher Staatsangehörigkeit ${ }^{2}$ und damit Ausländer im Sinne von $\S 2$ Abs. 1 AufenthG. Ausländische Gefangene kann beziehungsweise muss die Ausländerbehörde unter bestimmten Voraussetzungen in das Land ausweisen, dessen Staatsangehörigkeit sie besitzen. Die für den Erlass einer Ausweisungsverfügung zentralen Vorschriften finden sich in $\S \S 53,54$ und 55 AufenthG. Die bei Unionsbürgern, türkischen Arbeitnehmern mit Schutz des Beschlusses 1/80 des Assoziationsrates EWG-Türkei (kurz: ARB 1/80) und Drittstaatern, die absehbar einen Anspruch auf (Wieder-)Einreise geltend machen können, zu beachtenden Besonderheiten können hier nicht näher ausgeführt werden ${ }^{3}$. Ich muss mich auf die stark verkürzende Aussage beschränken, dass nicht privilegierte Ausländer, die rechtskräftig zu einer Freiheitsstrafe ohne Bewährung verurteilt werden, in der Regel ausgewiesen werden ${ }^{4}$. Als Rechtsfolgen löst die Ausweisung unter

1 Statistisches Bundesamt, Strafvollzug - Demographische und kriminologische Merkmale der Strafgefangenen, Fachserie 10, Reihe 4.1, 2013, S. 10, abrufbar unter: https://www.destatis.de/D E/Publikationen/Thematisch/Rechtspflege/StrafverfolgungVollzug/Strafvollzug2100410137004. pdf?_blob=publicationFile (abgerufen am 18.12.2014).

2 Statistisches Bundesamt (Anm. 1), S. 13; nach § 2 Abs. 1 AufenthG ist Ausländer jeder, der nicht Deutscher im Sinne von Art. 116 Abs. 1 GG ist.

$3 \mathrm{Zu}$ den Besonderheiten bei Angehörigen dieser Personengruppen siehe Pfaff, ZAR 2006, $121 \mathrm{ff}$. sowie Jung, StV 2007, 106, $109 \mathrm{f}$.

4 Vgl. $§ 54$ Abs. 1 AufenthG.

*Kontaktperson: Erol Pohlreich, Wissenschaftlicher Mitarbeiter am Lehrstuhl für Strafrecht, Strafprozessrecht, Europäisches Strafrecht und Neuere Rechtsgeschichte (Prof. Dr. Martin Heger) an der Humboldt-Universität zu Berlin. 
anderem die Pflicht des Ausländers zur Ausreise ${ }^{5}$ und ein gesetzliches Verbot der Wiedereinreise sowie des Aufenthalts im Bundesgebiet aus ${ }^{6}$. Eine vollziehbare Ausweisungsverfügung kann, wo erforderlich, mit der Abschiebung ${ }^{7}$ des Gefangenen zwangsweise durchgesetzt werden.

Die aufenthaltsrechtliche Situation ausländischer Gefangener kann auch Folgen haben für den Strafvollzug - also das „Wie“ der Strafe - und die Strafvollstreckung - also das „Ob“ der Strafe. Ob und bejahendenfalls inwieweit in diesen Rechtsgebieten der Aufenthaltsstatus ausgewiesener Gefangener deren Ungleichbehandlung im Vergleich zu den Mitgefangenen hinreichend trägt, soll im Folgenden untersucht werden.

\section{Besonderheiten im Strafvollzug}

Den Strafvollzug beherrscht das Grundrecht des Strafgefangenen auf Resozialisierung. Dieses verpflichtet den Staat nicht nur, den Strafvollzug auf das Ziel auszurichten, dem Inhaftierten ein zukünftiges straffreies Leben in Freiheit zu ermöglichen ${ }^{8}$. Zusätzlich hierzu verpflichtet es den Staat, Maßnahmen zu ergreifen, um insbesondere Gefangene, die sich seit langem in Haft befinden, vor haftbedingter Depravation zu bewahren9. Der Erfüllung beider Pflichten dienen auch Hafterleichterungen in Form von Ausführungen, bei denen der Gefangene unter Aufsicht die Anstalt verlässt, und Ausgängen, bei denen der Gefangene unbeaufsichtigt eine bestimmte Zeit außerhalb der Anstalt verbringt ${ }^{10}$.

\section{Vollzugspraktische Schlechterstellung ausländischer Gefangener}

Das Strafvollzugsgesetz ${ }^{11}$ enthält keinen ausdrücklichen Anhaltspunkt dafür, dass bei der Entscheidung über Hafterleichterungen ausländische Gefangene im

$5 \S 51$ Abs. 1 Nr. 5, § 50 Abs. 1, § 84 Abs. 2Satz 1 AufenthG.

$6 \S 11$ Abs. 1 Satz lund 2 AufenthG.

$7 \S \S 58 \mathrm{ff}$. AufenthG.

8 BVerfGE 35, 202, 235f.; 36, 174, 188; 45, 187, 239; 96, 100, 115; 116, 69, 85 f.; stRspr.

9 Vgl. BVerfGE 45, 187, 238; 64, 261, 277; 98, 169, 200; 109, 133, $150 \mathrm{f}$.

10 Vgl. BVerfG StV 2011, 488, 490; R\&P 2012, 222, 224; BVerfG, Beschlüsse der 3. Kammer des Zweiten Senats vom 26.10.2011 - 2 BvR 1539/09 und vom 23.05.2013 - 2 BvR 2129/11.

11 Das Strafvollzugsgesetz ist ein Bundesgesetz und gilt nur noch in einigen Bundesländern. Infolge der am 21. September 2006 in Kraft getretenen, allgemein als „Föderalismusreform I“ bezeichneten Reform des GG ist die Gesetzgebungszuständigkeit für den Vollzug von Straf- und 
Vergleich zu ihren deutschen Mitgefangenen anders zu behandeln wären. Vollzugslockerungen in Form von Ausführungen oder Ausgängen (§ 11 Abs. 1 Nr. 2 StVollzG) dürfen gemäß $\S 11$ Abs. 2 StVollzG nur angeordnet werden, wenn der Gefangene zustimmt und wenn nicht zu befürchten ist, dass der Gefangene sich dem Vollzug der Freiheitsstrafe entziehen oder die Vollzugslockerungen zu Straftaten missbrauchen wird.

Die Vollzugspraxis berücksichtigt demgegenüber durchaus den aufenthaltsrechtlichen Status des Gefangenen. Ein Blick in die bundeseinheitlichen Verwaltungsvorschriften zum Strafvollzugsgesetz verdeutlicht dies: Danach ist Ausgang ausgeschlossen bei Gefangenen, gegen die Untersuchungs-, Auslieferungsoder Abschiebehaft angeordnet ist ${ }^{12}$. Dasselbe gilt für Gefangene, gegen die eine vollziehbare Ausweisungsverfügung für den Geltungsbereich des Strafvollzugsgesetzes besteht und die aus der Haft abgeschoben werden sollen ${ }^{13}$, wobei in diesem Fall Ausnahmen, die Zustimmung der Aufsichtsbehörde und das Benehmen mit der zuständigen Ausländerbehörde vorausgesetzt, zulässig sind ${ }^{14}$. Ähnliche Verwaltungsvorschriften gibt es auch zu anderen Hafterleichterungen wie beispielsweise Urlaub oder Ausgang aus wichtigem Anlass ${ }^{15}$.

In diesen Verwaltungsvorschriften manifestiert sich eine durch Beobachtungen der Vollzugspraxis ${ }^{16}$ bestätigte Tendenz dahin, dass Ausgänge für Gefange-

Untersuchungshaft vom Bund auf die Länder übergegangen; gemäß Art. 125a Abs. 1 GG gilt das frühere Strafvollzugsgesetz in einem Bundesland solange fort, bis dieses ein eigenes Gesetz zur Regelung des Strafvollzugs erlässt. Aus Gründen der Übersichtlichkeit wird hier die Frage der Resozialisierung ausländischer Gefangener am Beispiel der Vorschriften des Strafvollzugsgesetzes diskutiert. Näher zur Frage der Gesetzgebungskompetenz in Vollzugsangelegenheiten Pohlreich, in: Brunhöber/Höffler/Kaspar/Reinbacher/Vormbaum, Strafrecht und Verfassung, 2012, S. $117 \mathrm{ff}$.

12 Vgl. VV Nr. 6 Abs. 1 Buchstabe b) zu § 11 StVollzG.

13 Vgl. VV Nr. 6 Abs. 1 Buchstabe c) zu $§ 11$ StVollzG.

14 VV Nr. 6 Abs. 2 Satz 3 zu § 11 StVollzG. Teilweise wird das „Benehmen“ irrig mit „Einvernehmen“ gleichgesetzt, vgl. die Nachweise aus der Rspr. und Literatur bei Schmidt, Verteidigung von Ausländern, 3. Aufl. 2012, Rdn. 614.

15 Hierzu näher Laubenthal, Strafvollzug, 7. Aufl. 2015, Rdn. 334. Vereinzelt wird im Schrifttum gar die Auffassung vertreten, die Verwaltungsvorschriften zum Strafvollzugsgesetz, die die Gewährung von Resozialisierungsmaßnahmen für ausgewiesene Strafgefangene einschränken, würden „die gesetzlichen Zielsetzungen des Strafvollzugs nicht nur rechtswidrig unterlaufen, sondern auch subversiv korrigieren“; insofern verstießen sie gegen das Resozialisierungsgebot, vgl. Knödler, in: Cornel/Kawamura-Reindl/Maelicke/Sonnen, Resozialisierung, 3. Aufl. 2009, S. 422(426).

16 Hierzu J. Walter, NKrimPol 2007, 127, 130; Knödler, in: Cornel/Kawamura-Reindl/Maelicke/ Sonnen (Anm. 15), S. 422, 425 ff. m.w. N.; Tzschaschel zufolge kommen ausländische Gefangene erkennbar seltener in den Genuss von Ausgängen beziehungsweise Ausführungen als ihre deutschen Mitgefangenen. Von den jeweils 100 deutschen und ausländischen Gefangenen aus ihrer 
ne, gegen die eine vollziehbare Ausweisungsverfügung besteht und die aus der Haft abgeschoben werden sollen, eher die Ausnahme sind. Solche Gefangene sind in der Praxis des Strafvollzugs gegenüber ihren deutschen Mitgefangenen benachteiligt. Am üblichen, d.h. bei deutschen Gefangenen zum Tragen kommenden Behandlungsprogramm können im Allgemeinen offenbar nur die ausländischen Gefangenen partizipieren, bei denen entweder die Ausländerbehörde ausdrücklich von einer Ausweisung abgesehen hat oder bei denen von vornherein nicht zwingend mit einer Ausweisung beziehungsweise Abschiebung zu rechnen ist ${ }^{17}$.

Umgekehrt, so wird berichtet, sehen Vollzugsanstalten von der Bewilligung von Lockerungen bei an sich prognostisch als günstig Einzustufenden schon dann ab, wenn die Möglichkeit einer Ausweisung besteht. Die Ausländerbehörden entschieden nämlich oft erst während bestehender Haft - bisweilen erst gegen Ende der Haftstrafe ${ }^{18}$ - über die Frage der Ausweisung des Verurteilten; beschreite dieser gegen eine für ihn nachteilige Entscheidung der Ausländerbehörde den Rechtsweg, ziehe dies die ohnehin schon lang anhaltende Ungewissheit über aufenthaltsrechtliche Maßnahmen weiter in die Länge mit der Konsequenz, dass die Vollzugsanstalt zur Sicherstellung einer eventuellen Ausweisung von Lockerungen absehe $^{19}$.

Diese für die Resozialisierung ausgewiesener Strafgefangener nachteilige Vollzugspraxis scheint auch im Schrifttum Anhänger zu finden. Einige Autoren erachten, nimmt man sie beim Wort, Resozialisierung in Fällen drohender Abschiebung oder auch schon bei anhängigem Abschiebeverfahren vielfach als ,wenig sinnvoll“20, „nicht sinnvoll“21 oder gar „sinnlos“22. Dementsprechend nimmt es kaum Wunder, dass teilweise der Standpunkt vertreten wird, bei ausländischen Gefangenen, denen auch nur die Ausweisung drohe, genüge wohl eine Verwahrung ${ }^{23}$.

Studie wurde 22 deutschen Gefangenen Ausgang beziehungsweise eine Ausführung genehmigt, während der entsprechende Anteil bei den ausländischen Gefangenen bei $11 \%$ lag, siehe TzSchaschel, Ausländische Gefangene im Strafvollzug, 2002, S. 58.

17 Tzschaschel (Anm. 16), S. $117 \mathrm{f}$.

18 Rothaus, ZfStrVo 1992, 41, 43.

19 Dünkel/Kunkat, NKrimPol 1997, 24, 29; M. Walter, Strafvollzug, 2. Aufl. 1999, S. 312; Winchenbach, psychosozial 65 (1996), S. 7, 13.

20 Meyer-Goßner/Schmitt, StPO, 58. Aufl. 2015, § 456a Rdn. 1; Groß, StV 1987, 36.

21 OLG Hamm BeckRS 2006 Nr. 03447; OLG Frankfurt a. M. NStZ-RR 2001, 93, 94.

22 Boese, Ausländer im Strafvollzug, 2003, S. 231, 245.

23 Finkbeiner/Karsten/Meiners, ZfStrVo 1993, 343; Koepsel, ZfStrVo 1983, 200, 201; ders., in: Bundeskriminalamt (Hrsg.), Moderne Sicherheitsstrategien gegen das Verbrechen, 1999, S. 249, 260. 


\section{Resozialisierung ausländischer Gefangener bei (drohender) Ausweisung}

Also nur ein Verwahrvollzug - allenfalls durch Teilnahme an Sport- und Fernsehgruppen und Gesprächskontakte mit Landsleuten verziert ${ }^{24}$ - für ausländische Gefangene mit Ausweisungsverfügung? Haben solche Gefangene keine realistische Aussicht auf Ausgang?

\section{a) Geltung des Resozialisierungsgebots für alle Gefangenen}

In der Tat kann man sich fragen, was das verfassungsrechtliche Resozialisierungsgebot bei ausgewiesenen und somit solchen Gefangenen, die nach der Haftverbüßung wohl nicht mehr in Deutschland leben werden, erfordert.

Begreift man Resozialisierung als Wiedereingliederung des Gefangenen in die deutsche Gesellschaft, erscheint es zumindest nicht ganz ausgeschlossen, ausgewiesenen Gefangenen ein Recht auf hierauf zielende Maßnahmen abzusprechen. Mangels Aussicht dieser Gefangenen, sich nach Haftentlassung rechtmäßig im Bundesgebiet aufhalten zu dürfen, erscheint ihr Interesse an einer Wiedereingliederung in die deutsche Gesellschaft auf den ersten Blick kaum anerkennenswert. Für die Anerkennung dieses Interesses lässt sich indes ins Feld führen, dass die Betroffenen ohne Resozialisierungsmaßnahmen möglicherweise eher illegal in die Bundesrepublik zurückkehren könnten oder im Ausland Straftaten begehen könnten, die den „deutschen Rechtskreis“ berühren²5. Abgesehen davon müssen selbst ausgewiesene Strafgefangene sich auf das Resozialisierungsgebot in seiner Ausprägung als Wiedereingliederungsgebot berufen können, da man auch einem deutschen Gefangenen mit Auswanderungsabsicht wohl kaum schon wegen dieser Absicht Resozialisierungsmaßnahmen verwehren würde.

Dies schließt nicht aus, ausgewiesenen Gefangenen besondere Maßnahmen für das Land, in dem sie nach Haftentlassung leben werden, zukommen zu lassen. Ob die Anstalt allerdings kraft verfassungsrechtlichen Auftrags darauf festgelegt ist, für jeden Gefangenen ein spezifisches Wiedereingliederungsprogramm für das Land, in dem er nach Haftentlassung leben wird, zu entwickeln, erscheint zweifelhaft. Denn bei der Beurteilung der Frage, welche Mittel die Vollzugsanstalt zur Erreichung des Resozialisierungsgebots einzusetzen hat, sind nicht nur die für den

24 In solchen Maßnahmen wird teilweise die gesamte Behandlung gesehen, die ausländischen Gefangenen angeboten werden kann, vgl. Koepsel, ZfStrVo 1983, 200, 201.

25 Ebenso Giehring, Festschrift 125 Jahre Staatsanwaltschaft Schleswig-Holstein, 1992, S. 469, 490. 
Strafgefangenen streitenden Belange, sondern auch die der Anstalt entstehenden Schwierigkeiten zu berücksichtigen ${ }^{26}$, und es bescherte den Haftanstalten zweifellos kaum zu bewältigende Probleme, wenn für die Vielzahl der Länder, in denen die Gefangenen nach Haftentlassung leben, ein jeweils eigenes Wiedereingliederungsprogramm zu entwickeln und dieses womöglich fortwährend an die sich ändernden Bedingungen im jeweiligen Land anzupassen wäre. Andererseits wird die Vollzugsanstalt nicht ihre Augen davor verschließen dürfen, wenn ausgewiesene Gefangene ihr gegenüber auf Besonderheiten in ihrer Heimat hinweisen, und dem Begehren solcher Gefangener auf Durchführung von Maßnahmen, die die Besonderheiten der Heimat der Gefangenen berücksichtigen, Folge geben müssen, wenn dies auch in Ansehung des hiermit verbundenen Aufwands der Anstalt zumutbar ist. Dabei wird mit steigender Zahl der Gefangenen mit vergleichbarem Hintergrund eher von einer Zumutbarkeit - und spiegelbildlich hierzu von einer gesteigerten Rechtfertigungslast der Vollzugsanstalt, will sie gleichwohl von der Entwicklung beziehungsweise Durchführung länderspezifischer Maßnahmen absehen - auszugehen sein.

Jedenfalls steht das Resozialisierungsgebot in seiner Ausprägung, dass es zur Verhinderung von haftbedingter Depravation verpflichtet, einem schlichten Wegsperren ausgewiesener Gefangener ohne Behandlung, also einem Verwahrvollzug, entgegen. Auch das Bundesverfassungsgericht hat in der Versagung jeglicher Lockerungen unter bloßem Hinweis auf ein anhängiges Ausweisungsverfahren die Gefahr erkannt, dass Strafhaft in unzulässiger Weise zur Abschiebehaft umfunktioniert und der Strafvollzug für nichtdeutsche Verurteilte zum bloßen Verwahrvollzug würde, der mit dem Resozialisierungsgebot nicht vereinbar wäre ${ }^{27}$. Das Resozialisierungsgebot gilt nach der Rechtsprechung des Bundesverfassungsgerichts auch für Strafgefangene, die aus der Haft heraus abgeschoben werden sollen, weil es nicht allein dem (inner)staatlichen Interesse an einer künftigen Straffreiheit des Verurteilten dient, sondern vor allem auch dessen Grundrechte schützt ${ }^{28}$. Auf das verfassungsrechtliche Resozialisierungsgebot kann sich also jeder Strafgefangene berufen, und zwar unabhängig von seinem

26 Vgl. BVerfGK 20, 307, 313.

27 BVerfG ZfStrVo 2002, 372, 373; BVerfG, Beschluss der 3. Kammer des Zweiten Senats vom 29.01.2004 - 2 BvR 2167/03; vgl. auch BVerfG, Beschluss der 3. Kammer des Zweiten Senats vom 15.03.2004 - 2 BvR 1530/03. Aus der fachgerichtlichen Rechtsprechung vgl. statt vieler OLG Stuttgart StraFo 2004, 326.

28 BVerfG, Beschluss der 2. Kammer des Zweiten Senats vom 10. Oktober 2012 - 2 BvR 2025/12. 
aufenthaltsrechtlichen Status ${ }^{29}$ und dem Aufenthaltsort nach seiner Haftentlassung.

\section{b) Anforderungen an die Vollzugspraxis}

Dieses Verständnis des Resozialisierungsgebots befindet sich nur scheinbar in einem unlösbaren Konflikt mit den oben dargestellten Verwaltungsvorschriften, die das Spektrum auf Wiedereingliederung ausgewiesener Gefangener abzielender Behandlungsmaßnahmen im Vergleich zu ihren Mitgefangenen doch erheblich zu reduzieren scheinen. Der Widerspruch löst sich auf, wenn man sich die Funktion und Bindungswirkung von Verwaltungsvorschriften vergegenwärtigt.

Verwaltungsvorschriften sollen der Vollzugsbehörde bei Einzelfallentscheidungen helfen und gewährleisten, dass die Behörde ihr Ermessen gleichförmig ausübt. Die mit ihnen getroffene Selbstbindung der Verwaltung muss aber ihrerseits verfassungskonform sein. Sie enthebt die Behörde außerdem nicht von ihrer Verpflichtung, im Einzelfall zu überprüfen, ob die Voraussetzungen vorliegen, für die die Selbstbindung gedacht war $^{30}$. Dementsprechend ist in der fachgerichtlichen Rechtsprechung anerkannt, dass Vollzugslockerungen wegen eines anhängigen Ausweisungsverfahrens nicht pauschal wegen Flucht- oder Missbrauchsgefahr versagt werden dürfen ${ }^{31}$.

Die Verwaltungsvorschriften zum Strafvollzugsgesetz sind also Anfangsvermutungen und Entscheidungshilfen, die die Vollzugsbehörde nicht ihrer Pflicht zur Einzelfallprüfung entheben. Unterlässt die Behörde aus Richtlinienhuberei die Prüfung von Einzelfallmomenten und belässt es bei der starren Anwendung von Verwaltungsvorschriften, widerspricht ihre Entscheidung den gesetzlichen Anforderungen $^{32}$. Denn die Vollzugsbehörde ist gehalten, die für die Beurteilung maßgeblichen Gesichtspunkte einzelfallbezogen darzustellen und abzuwä-

29 BVerfGE 96, 100, 115; vgl. auch Di Fabio, in: Maunz/Dürig, GG, Art. 2 Rdn. 216 (Juli 2001); Leyendecker, (Re-) Sozialisierung und Verfassungsrecht, 2002, S. 151.

30 Vgl. BVerfGK 12, 210, 219.

31 OLG Frankfurt a. M. ZfStrVo 1983, 249, 250; NStZ 1983, 93; ZfStrVO 1991, 372, 373; OLG Celle ZfStrVO 1984, 251 f.; Neubacher, in: Laubenthal/Nestler/Neubacher/Verrel, Stafvollzugsgesetze, 12. Aufl. 2015, Abschnitt B Rdn. 48; Ullenbruch, in: Schwind/Böhm/Jehle/Laubenthal, StVollzG, 6. Aufl. 2013, § 13 Rdn. 17; Laubenthal, Strafvollzug (Anm. 15), Rdn. 334.

32 Vgl. OLG Koblenz ZfStrVo 1978, 123, 124; OLG Frankfurt a. M. NJW 1978, 334f.; OLG Celle JR 1978, 258, 259; OLG Nürnberg ZfStrVo SH 1978, 50, 51; OLG Hamburg NStZ 1981, 237f.; OLG Schleswig BeckRS 2007 Nr. 02371; vgl. auch Lesting in: Feest/Lesting, StVollzG, 6. Aufl. 2012, § 11 Rdn. 40; Laubenthal, in: Laubenthal/Nestler/Neubacher/Verrel (Anm. 31), Abschnitt E Rdn. 150. 
gen $^{33}$. Zwar mag den genannten Verwaltungsvorschriften zu $\S 11$ StVollzG die allgemeine Erfahrung zugrunde liegen, dass bei ausgewiesenen Gefangenen eine der Genehmigung von Vollzugslockerungen entgegenstehende Fluchtgefahr besteht ${ }^{34}$. Die Vollzugsbehörde muss gleichwohl den Einzelfall dahin überprüfen, ob Gesichtspunkte vorliegen, die diese allgemeine Erfahrung widerlegen. Wenn auch dann noch die Gewährung von Ausgang ausscheidet, muss sie Ausführungen erwägen und hierbei insbesondere berücksichtigen, dass der Gefangene bei Ausführungen von Vollzugsbediensteten beaufsichtigt wird. Darüber hinaus kann, wo trotz Begleitung durch Vollzugsbedienstete erforderlich, einer Fluchtgefahr dadurch entgegengewirkt werden, dass der Gefangene während der Ausführungen gefesselt ist oder die Vollzugsbediensteten bewaffnet sind. Den Verzicht auf eine konkrete Entscheidungsbegründung, die über den bloßen Hinweis auf das anhängige Ausweisungsverfahren hinausgeht, ist allenfalls dann vertretbar, wenn nach den Einzelfallumständen ein Missbrauch der Vollzugslockerungen mit Blick auf die drohende Abschiebung offensichtlich vorauszusehen wäre ${ }^{35}$.

\section{Besonderheiten in der Strafvollstreckung}

Im Strafvollstreckungsrecht werden rechtskräftig ausgewiesene Gefangene gegenüber anderen Gefangenen zunächst bessergestellt: Während bei allen Gefangenen mit günstiger Kriminalprognose nach Verbüßung von zwei Dritteln einer zeitigen Freiheitsstrafe die Vollstreckung des Strafrests zur Bewährung ausgesetzt werden kann $^{36}$, gilt für aufenthaltsrechtlich nicht privilegierte ausländische Strafgefangene eine Besonderheit.

\section{Verfahren nach $\S 456$ a StPo}

Nach § 456a Abs. 1 StPO kann die Vollstreckungsbehörde von der Vollstreckung einer Freiheitsstrafe, einer Ersatzfreiheitsstrafe oder einer Maßregel der Besserung

33 Vgl. OLG Frankfurt a. M. NJW 1978, 334, 335; NStZ-RR 2004, 94; OLG Stuttgart StraFo 2004 , 326; OLG Karlsruhe NStZ-RR 2007, 325; OLG Koblenz NStZ-RR 2008, 190.

34 A. A. Feest/Graebsch, in: Feest/Lesting (Anm. 32), Anh § 175 Rdn. 18, denen zufolge es jeglicher Grundlage entbehre, dass eine Ausweisungsverfügung als Prognosekriterium für Fluchtgefahr tauge.

35 BVerfG StV 2003, 677, 678. Vgl. auch BVerfG NStZ-RR 2012, 387, 388.

$36 \S 57$ Abs. 1 Satz 1 StGB. 
und Sicherung absehen, wenn der Betroffene aus dem Bundesgebiet ausgewiesen wird. Wann die Vollstreckungsbehörde hierüber entscheidet, ist von Bundesland zu Bundesland unterschiedlich. Regelmäßig sehen die Richtlinien der Länder eine Prüfung spätestens zum Halbstrafenzeitpunkt vor ${ }^{37}$. Damit können nichtdeutsche Strafgefangene, gegen die eine vollziehbare Ausweisungsverfügung vorliegt, noch vor dem Zeitpunkt, in dem eine Reststrafenaussetzung frühestens in Betracht käme, aus der Haft entlassen werden ${ }^{38}$.

\section{a) Normzweck und Entscheidungspraxis}

Die Vorschrift geht auf das Deutsche Auslieferungsgesetz aus dem Jahr $1929^{39}$ zurück. Der historische Gesetzgeber erachtete es „ausnahmsweise“ für mit den Interessen der inländischen Rechtspflege vereinbar, von der Strafvollstreckung abzusehen, „um die alsbaldige Auslieferung des ausländischen [...] Verurteilten zu ermöglichen“ “40. Mit der Zeit wurde die Möglichkeit der Reststrafenaussetzung nach §456a StPO für die Senkung von Vollstreckungskosten, also für justizökonomische Zwecke, fruchtbar gemacht ${ }^{41}$. Einem Teil der Literatur zufolge besteht die Ratio der Vorschrift zudem im Fehlen individualpräventiver Bedürfnisse in den von der Vorschrift betroffenen Fällen und darin, dass ausgewiesene Gefangene besonders belastenden Haftbedingungen ausgesetzt sei-

37 Appl, in: Karlsruher Kommentar zur StPO, 7. Aufl. 2013, § 456a Rdn. 3a. Eine Übersicht zu den Richtlinien der Länder findet sich bei Schmidt (Anm. 14), Rdn. 582ff. Die einschlägigen Verwaltungsrichtlinien der Länder sind im Wortlaut abrufbar unter http://www.verteidigung-von-aus laendern.de (abgerufen am 18.12.2014).

38 Hiermit korreliert auch die bis zur rechtskräftigen innerstaatlichen Erledigung des Strafverfahrens bestehende Möglichkeit der Verfahrenseinstellung nach $\S 154$ b Abs. 1, 3 StPO für den Fall, dass der Beschuldigte wegen der Tat einer ausländischen Regierung ausgeliefert wird oder aus dem Geltungsbereich der StPO ausgewiesen wird.

39 RGBl. IS. 239.

40 Vgl. RT-Drucks. 4/362 S. 15.

41 Vgl. BT-Drucks. 10/2720 S. 16; Groß, StV 1987, 36; Giehring, Festschrift 125 Jahre Staatsanwaltschaft Schleswig-Holstein, S. 469, 480; Meyer-Goßner/Schmitt (Anm. 20), § 456a Rdn. 1; das OLG Hamm NStZ 1983, 524 formuliert dies besonders drastisch: „Denn der Gesetzgeber hat durch die Einführung des § 456a StPO die Möglichkeit, bei Ausländern von der Strafvollstreckung abzusehen, nicht im Interesse des Ausländers geschaffen, um diesen gegenüber deutschen Strafgefangenen zu begünstigen. Vielmehr sollte im öffentlichen Interesse unter Durchbrechung des grundsätzlich auch für die Vollstreckung geltenden Legalitätsprinzips den deutschen Strafverfolgungsbehörden die Möglichkeit geschaffen werden, die inländischen Stellen beizeiten von der Last der Vollstreckung von Strafen gegen Ausländer befreien zu können.“ 
$\mathrm{en}^{42}$ und dies durch ein Absehen von der weiteren Vollstreckung ausgeglichen werden solle ${ }^{43}$.

Vom Verfahren nach $\S 456$ a Abs. 1 StPO wird bei einem beachtlichen Teil der ausländischen Gefangenen Gebrauch gemacht ${ }^{44}$. Die Zahl der Verfahren nach dieser Vorschrift wird zwar nicht statistisch erfasst. Jedoch ergab eine im Jahr 2002 veröffentlichte Untersuchung von 100 Gefangenenpersonalakten jeweils deutscher und ausländischer Gefangener, dass bei 71 von 84 ausländischen Gefangenen mit Ausweisungsverfügung ${ }^{45}$ nach $\S 456$ a Abs. 1 StPO von der weiteren Vollstreckung abgesehen wurde; dies entspricht einer beachtlichen Quote von $86,6 \%{ }^{46}$. Von diesen 71 Gefangenen wiederum wurden 67 Gefangene aus der Haft abgeschoben ${ }^{47}$.

\section{b) Möglichkeit der Vollstreckungsnachholung bei Wiedereinreise}

Ein Absehen von der weiteren Vollstreckung nach § 456a StPO hat nur vorläufigen Charakter. Kehrt der Verurteilte nach Deutschland zurück, droht ihm die Inhaftierung und die Nachholung der Vollstreckung ${ }^{48}$. Um die Nachholung sicherzustellen, kann die Vollstreckungsbehörde neben dem Absehen von der weiteren Vollstreckung einen Vollstreckungshaftbefehl erlassen ${ }^{49}$. Da das verfassungsrechtliche Gebot einer funktionstüchtigen Strafrechtspflege ${ }^{50}$ im Fall einer Einreise des Verurteilten regelmäßig eine - strafrechtlich durch $\S 258$ a StGB abgesicherte - Voll-

42 Insoweit ist indes daran zu erinnern, dass bei der Normauslegung prinzipiell von einer korrekten und fairen Anwendungspraxis in einer freiheitlich-demokratischen Demokratie auszugehen ist (vgl. BVerfGE 30, 1, 27; 63, 45, 64), die in der Vollzugspraxis zu beobachtende Härte gegenüber ausgewiesenen Gefangenen nach dem oben Gesagten den gesetzlichen Anforderungen aber nicht genügt. Härten, die rechtsstaatlichen Maßstäben nicht standhalten, sind nicht im Wege einer breiten Anwendung von $\S 456$ a StPO durch die Vollstreckungsbehörde, sondern im Wege gerichtlicher Kontrolle vollzugsbehördlicher Entscheidungen zu korrigieren.

43 Groß, StV 1987, 36. Zu diesen Gesichtspunkten ausführlich Giehring, Festschrift 125 Jahre Staatsanwaltschaft Schleswig-Holstein, S. 469, $483 \mathrm{ff}$.

44 Die Vorschrift ist übrigens, nachdem Art. 16 Abs. 2 Satz 2 GG die Auslieferung Deutscher zulässt, auch auf diese anwendbar, vgl. BVerfG, Beschluss der 3. Kammer des Zweiten Senats vom 09.10.2003 - 2 BvR 1497/03, NJW 2004, 356.

45 Tzschaschel (Anm. 16), S. 44.

46 Tzschaschel (Anm. 16), S. 92.

47 Tzschaschel (Anm. 16), S. 102.

$48 \S 456$ a Abs. 2Satz 1 StPO.

$49 \S 456$ a Abs. 2 Satz 3 StPO.

50 Vgl. BVerfGE 33, 367, 383; 46, 214, 222; 122, 248, 272; 130, 1, 26; 133, 168, 199. 
streckungspflicht der Vollstreckungsbehörde begründet ${ }^{51}$, muss der Verurteilte im Fall einer Einreise nach Deutschland mit seiner Inhaftierung rechnen, und zwar auch dann, wenn die Ausländerbehörde - etwa aufgrund familiärer Bindungen des Verurteilten nach Deutschland - die Wirkungen der Ausweisung befristet und der Verurteilte nach Fristablauf wieder einreist; die Vollstreckungsbehörde kann die Vollstreckung der Reststrafe in jedem Fall der Wiedereinreise nachholen, also unabhängig davon, ob der Verurteilte sich nach seiner Wiedereinreise aufenthaltsrechtlich legal oder illegal im Bundesgebiet aufhält ${ }^{52}$.

Vor diesem Hintergrund erachten einzelne Stimmen in der Literatur die Vorschrift des § 456a StPO teilweise für nicht mehr zeitgemäß, weil ihr das Bild von ausländischen Straftätern als „fahrende Gesellen“ zugrunde liege, viele dieser Straftäter allerdings in Deutschland mehr oder weniger verwurzelt seien und durch das Bestehen eines Vollstreckungshaftbefehls daran gehindert würden, nach Ablauf der Frist für die Wirkungen der Ausweisung wieder in das Bundesgebiet einzureisen ${ }^{53}$.

\section{Möglichkeit einer Reststrafenaussetzung}

Das Absehen von der weiteren Vollstreckung kann sich für den Betroffenen, obgleich prima facie nur begünstigend, als Danaergeschenk erweisen, wenn ihm - etwa aufgrund enger Bindungen mit hierzulande Ansässigen - an einem Aufenthalt im Bundesgebiet besonders gelegen ist. Denn mangels in der Absehensanordnung selbst liegender Beschwer für ihn - erst die anschließende Auslieferung, Ausweisung oder Abschiebung beschwert den Betroffenen - kann der Verurteilte die Anordnung des Absehens von der weiteren Vollstreckung nicht anfechten $^{54}$. Befindet er sich nach einem Vorgehen nach § 456a StPO im Ausland und will er wieder nach Deutschland zurückkehren, ohne den Strafrest verbüßen zu müssen, könnte er zwar einen Antrag auf Reststrafenaussetzung nach $§ 57$

51 OLG Karlsruhe ZfStrVo 1997, 369, 370; OLG Frankfurt a. M. NStZ-RR 2001, 93, 94; vgl. auch OLG Hamburg NStZ-RR 1999, 123, 125.

52 Feest/Graebsch, in: Feest/Lesting (Anm. 32), Anh § 175 Rdn. 33.

53 Vgl. Pfaff, ZAR 2006, 121.

54 Vgl. OLG Frankfurt a. M. NStZ-RR 1999, 126, 127; OLG Celle NStZ 2008, 221, 222; OLG Karlsruhe NStZ 2008, 222; OLG Stuttgart, Beschluss vom 04.02.2014 - 4 VAs 1/13; Meyer-Goßner/Schmitt (Anm. 20), § 456a Rdn. 9; Paeffgen, in: Systematischer Kommentar zur StPO, 4. Aufl. 2013, § 456a Rdn. 9; Appl, in: KK StPO (Anm. 37), § 456a Rdn. 5; Pfeiffer, StPO, 5. Aufl. 2005, § 456a Rdn. 5; a. A. Pfaff, ZAR 2006, 121, 124. 
StGB stellen ${ }^{55}$. Allerdings gehört zum Reststrafenaussetzungsverfahren grundsätzlich ${ }^{56}$ eine mündliche Anhörung des Verurteilten und in einigen Fällen auch dessen kriminalprognostische Begutachtung ${ }^{57}$. Nimmt der Verurteilte aber an diesen Verfahrensschritten teil, setzt er sich der Gefahr der Reststrafenvollstreckung aus.

Jenseits der gesetzlich genannten Ausnahmefälle ist ein Absehen von der Durchführung einer mündlichen Anhörung unzulässig. Das von der Gegenmeinung $^{58}$ vorgebrachte Argument, dass Gerichte nach den Gesetzgebungsmaterialien auf eine mündliche Anhörung dort, wo ihre Durchführung eine bloße Formalie wäre, verzichten sollten, verfängt nicht. Für die Gesetzesauslegung maßgeblich ist nämlich der in der Norm zum Ausdruck kommende objektivierte Wille des Gesetzgebers, wie er sich aus dem Wortlaut und dem Sinnzusammenhang der Vorschrift ergibt ${ }^{59}$. Der Entstehungsgeschichte einer Vorschrift kommt für deren Auslegung nur insofern Bedeutung $\mathrm{zu}$, als sie die Richtigkeit einer nach den angegebenen Grundsätzen erhaltenen Auslegung bestätigt oder Zweifel behebt, die auf dem angegebenen Weg allein nicht ausgeräumt werden können ${ }^{60}$. Der Wille des Gesetzgebers kann nur insoweit berücksichtigt werden, als er in dem Gesetz selbst einen hinreichend bestimmten Ausdruck gefunden hat ${ }^{61}$. Der Gesetzeswortlaut des $§ 454$ StPO ist aber, was die Zulässigkeit des Verzichts auf eine mündliche Anhörung angeht, eindeutig: Anders als die übrigen Verfahrensbeteiligten im Reststrafenaussetzungsverfahren, die nur zu hören sind, ist der Verurteilte nach § 454 Abs. 1 Satz 3 StPO mündlich zu hören. Von der so in ihrer Bedeutung hervorgehobenen obligatorischen mündlichen Anhörung des Verurteilten wollte der Gesetzgeber nur in den abschließend in Satz 4 der Vorschrift enumerierten Fällen eine

55 Ein Absehen von Strafe nach § 456a StPO steht der Durchführung eines Reststrafenaussetzungsverfahrens nicht entgegen, vgl. Baier, in: Radtke/Hohmann, StPO, 2011, § 456a Rdn. 4 m.w. N. aus der Rechtsprechung.

56 Teilweise wird die Durchführung der Anhörung für zwingend erachtet, vgl. OLG Schleswig, Beschluss vom 24.12.2003 - 2 Ws 329/03; OLG Bremen NStZ 2010, 718: „Dem auf die persönliche Anhörung des Verurteilten gestützten Eindruck der StVK kommt bei der nach $§ 57$ Abs. 1 StGB vorzunehmenden Sozialprognose wesentliche Bedeutung zu“. A. A. OLG Düsseldorf NStZ 2000, 333; Beschlüsse vom 19.12.1988 - 3 Ws 865/88, vom 09.01.1996 - 3 Ws 592/95; OLG Hamm, Beschluss vom 12. Februar 2008 - 3 Ws 61/08; OLG Köln, Beschluss vom 09.01.2009 - 2 Ws 644645/08; Meyer-Goßner/Schmitt (Anm. 20), § 454 Rdn. 32; Hubrach, in: Leipziger Kommentar, StGB, Band 2, 12. Aufl. 2008, § 57 Rdn. 83.

57 Vgl. § 454 Abs. 1 Satz 2 und 3, Abs. 2 Satz 1 StPO.

58 Vgl. statt vieler OLG Karlsruhe NJW 1976, 302.

59 BVerfGE 1, 299, 312; 11, 126, 130 f.; 105, 135, 157; 133, 168, 205; stRspr.

60 Vgl. BVerfGE 1, 299, 312; 8, 274, 307; 10, 234, 244; 48, 246, 256; 71, 81, 106; 105, 135, 157; stRspr. 61 Vgl. BVerfGE 11, 126, 130; 13, 261, 268; 47, 109, 127; 62, 1, 45; 105, 135, 157. 
Ausnahme zulassen. Für den abschließenden Charakter der ausdrücklich genannten Ausnahmefälle spricht zum einen, dass das Strafvollstreckungsgericht bei Vorliegen eines der in $\S 454$ Abs. 1 Satz 4 StPO genannten Fälle von einer mündlichen Anhörung des Verurteilten bloß absehen $k a n n^{62}$. Zum anderen verzichtete der Gesetzgeber bei der Aufzählung auf Formulierungen wie ,insbesondere“ oder „,beispielsweise“. Eine vom Gesetzgeber nicht genannte und gleichwohl anzuerkennende Ausnahme mag allenfalls in der von der Rechtsprechung ${ }^{63}$ entwickelten Fallgruppe anzunehmen sein, in der der Verurteilte eine mündliche Anhörung ausdrücklich und unmissverständlich ablehnt ${ }^{64}$; denn eine mündliche Anhörung des Verurteilten gegen seinen Willen ließe sich nicht erzwingen. In der hier diskutierten Konstellation sollte sich der Verurteilte freilich gut überlegen, ob er freiwillig dem Gericht seinen Verzicht auf die Durchführung einer mündlichen Verhandlung erklären will. Verzichtet der Verurteilte nämlich auf die Durchführung einer mündlichen Anhörung, begibt er sich hiermit einer Chance, aktiv dabei mitzuwirken, dass das Gericht die Voraussetzungen für eine Reststrafenaussetzung auf gründlicher Tatsachengrundlage prüfen und bejahen kann.

Zur Lösung des Dilemmas nahm das OLG Schleswig, die Durchführung einer Anhörung im Reststrafenaussetzungsverfahren für zwingend ansehend, eine gerichtliche Pflicht an, dem Verurteilten für die zur Anhörungswahrnehmung erforderliche An- und Abreise freies Geleit gegen Maßnahmen nach $\S 456$ a Abs. 2 StPO zu gewähren ${ }^{65}$. Dieser Lösungsweg vermag indes nicht zu überzeugen. Da das freie Geleit nach $\S 295$ StPO einen Beschuldigten nur vor Untersuchungshaft schützt, scheidet eine direkte Anwendung der Vorschrift aus ${ }^{66}$, so dass allenfalls ihre analoge Anwendung auf die hier diskutierte Konstellation in Betracht kommt. Eine analoge Anwendung von $§ 295$ StPO scheitert jedoch daran, dass hinter dem freien Geleit der Zweck steht, das staatliche Interesse an einer Beendigung des Strafverfahrens um der materiellen Gerechtigkeit willen zu verwirklichen ${ }^{67}$. Einge-

62 Kuckuk, NJW 1976, 815.

63 BGH NStZ 1995, 610 f.; NJW 2000, 1663; OLG Hamm MDR 1975, 775; MDR 1980, 870; NStZ 2011, S. 119; OLG Düsseldorf MDR 1981, 1039; NStZ 1987, 524; NStZ 1988, 243; NJW 1993, 1665, 1666; OLG Frankfurt a. M. NStZ-RR 1997, 28; NStZ-RR 2006, 357; OLG Karlsruhe NStZ 1996, 302.

64 Da das Grundrecht aus Art. 103 Abs. 1 GG dem Einzelnen nur ein Recht auf Äußerungsmöglichkeit, nicht aber eine Äußerungspflicht vermittelt (vgl. Schmidt-Aßmann, in: Maunz/Dürig, GG, Art. 103 Abs. 1 GG), liegt in der Anerkennung der Fallgruppe des vom Verurteilten erklärten Anhörungsverzichts kein Gehörsverstoß.

65 OLG Schleswig, Beschluss vom 24.12.2003 - 2 Ws 329/03.

66 Jung, StV 2007, 106, 108.

67 OLG Düsseldorf NStZ-RR 1999, 245. Für die so verstandene Zweckrichtung der Vorschrift spricht § 295 Abs. 3 Var. 1 StPO, wonach das sichere Geleit mit einem auf Freiheitsstrafe lautenden Urteil erlischt. 
denk dieser Zweckrichtung lassen sich die Situationen im gesetzlich geregelten und im hier diskutierten Fall nicht miteinander vergleichen.

Paeffgen $^{68}$ zufolge soll die Vollstreckungsbehörde von einer Nachholung der Vollstreckung absehen dürfen, wenn nach längerer Zeit das Vollstreckungsinteresse gemindert sei ${ }^{69}$ - etwa aufgrund mehrjährigen Aufenthalts im Bundesgebiet nach Rückkehr ${ }^{70}$ - oder der Verurteilte auf Veranlassung deutscher Behörden einreise $^{71}$. Von einer - hier allein in Betracht kommenden - behördlichen Veranlassung wird man aber nicht ernsthaft reden können, wenn der Verurteilte selbst das Verfahren nach $\S 454 \mathrm{StPO}$ anstrebt und die Initiative hierzu von ihm ausgeht.

Möglicherweise ist die Vollstreckungsbehörde aufgrund prozessualer Fürsorgepflichten gehalten, auf Antrag des Verurteilten den Vollstreckungshaftbefehl auszusetzen, wenn dieser eine Reststrafenaussetzung beantragt. Dies wird in der Literatur $^{72}$ vertreten für den Fall, dass die zuständige Ausländerbehörde dem Verurteilten eine zeitlich begrenzte Betretenserlaubnis für das Reststrafenaussetzungsverfahren erteilt und der Verurteilte nur durch persönliche Anwesenheit beim Anhörungstermin sein Recht auf rechtliches Gehör effektiv wahrnehmen kann. Die zweite Voraussetzung wird regelmäßig erfüllt sein. Denn die mündliche Anhörung des Verurteilten dient dem Zweck, dass sich das Gericht einen persönlichen Eindruck vom Verurteilten verschaffen $\mathrm{kann}^{73}$. Da - nicht nur ausländische, sondern auch viele deutsche - Gefangene typischerweise nach Bildungsstand, materiellen Ressourcen und Kommunikationsmöglichkeiten für den Umgang mit den Kompliziertheiten der Rechtsordnung nicht gut gerüstet $\operatorname{sind}^{74}$, erscheint die gesetzgeberische Entscheidung für eine obligatorische mündliche Verhandlung besonders sinnvoll; wenn Gefangene allein schriftliche Stellungnahmen abgeben könnten, würde dies dem Gericht keine gleichwertige Erkenntnisgrundlage vermitteln ${ }^{75}$. Zwar scheidet eine mündliche Anhörung des Verurteil-

68 Paeffgen, in: SK StPO (Anm. 54), § 456a Rdn. 8.

69 Paeffgen, in: SK StPO (Anm. 54), § 456a Rdn. 8 unter Hinweis auf OLG Karlsruhe MDR 1992, 885; vgl. auch OLG Frankfurt a. M. StV 1985, 23; OLG Köln MDR 1991, 276.

70 Vgl. hierzu etwa OLG Karlsruhe NStZ 1994, 254.

71 Paeffgen, in: SK StPO (Anm. 54), § 456a Rdn. 8; ähnlich Stöckel, in: Kleinkecht/Müller/Reitberger, KMR StPO, § 456 a Rdn. 14.

72 Jung, StV 2007, 106, 108.

73 Vgl. BT-Drucks. 7/550 S. 309; BVerfG NJW 1993, 2301, 2302f.; Siehe BGHSt. 28, 138, 141; BGH NStZ 1995, 610; NJW 2000, 1663; OLG Schleswig NJW 1975, 1131; OLG Hamm, Beschluss vom 27.01.2014 - III-1 Ws 600/13, 1 Ws 600/13; Rieß, JR 1976, 118; Bringewat, NStZ 1996, 17, 20.

74 Dies haben Gerichte bei der Auslegung der (auch) für den Rechtsschutz Gefangener geltenden Verfahrensvorschriften zu beachten, vgl. BVerfGK 10, 509, 516; BVerfG NStZ-RR 2013, 120, 121; 2014, 93, 94.

75 So auch Baier, in: Radtke/Hohmann (Anm. 55), § 454 Rdn. 16. 
ten durch ein ersuchtes Gericht im Ausland aus, weil die Anhörung der zur Entscheidung berufenen Strafvollstreckungskammer einen persönlichen Eindruck vom Verurteilten verschaffen soll ${ }^{76}$. Man kann die mündliche Anhörung nach der Rechtsprechung allerdings ausnahmsweise durch eine Videokonferenz ersetzen, wenn der Verurteilte hiermit einverstanden ist und die technischen Vorrichtungen ausreichen, damit das Gericht sich einen umfassenden Eindruck vom Verurteilten verschaffen $\mathrm{kann}^{77}$. Soweit das OLG Frankfurt mit Beschluss aus dem Jahr 2006 die Auffassung vertrat, eine solche Videokonferenz sei regelmäßig ungeeignet, dem Gericht den erforderlichen Eindruck zu verschaffen, und enge den Verurteilten in seinen Ausdrucksmöglichkeiten ein $^{78}$, erscheint dies angesichts der sich technisch stetig verbessernden Telekommunikationsmöglichkeiten immer zweifelhafter.

Solange die Rechtsprechung aber an der Auffassung des OLG Frankfurt festhält, wird der Verurteilte - abgesehen von seltenen Evidenzfällen - regelmäßig nach Deutschland einreisen müssen, um seine Chancen im Reststrafenaussetzungsverfahren zu verbessern. Dass er in der Regel zur Wahrnehmung seines rechtlichen Gehörs in diesem Verfahren nach Deutschland einreisen muss, begründet allerdings entgegen Jung keine Pflicht der Vollstreckungsbehörde, den Vollstreckungshaftbefehl auszusetzen. Das Risiko einer Inhaftierung für den Fall der Einreise hat der Verurteilte zu tragen. Denn zu der im Gesetz vorgesehenen mündlichen Anhörung des Verurteilten im Reststrafenaussetzungsverfahren gehört nicht die Verbürgung, dass der Verurteilte sich im Zeitpunkt dieser Anhörung in Freiheit befindet. Im Gegenteil, typischerweise befindet sich ein Verurteilter, der eine Reststrafenaussetzung anstrebt, zum Zeitpunkt der mündlichen Anhö-

76 OLG Düsseldorf NStZ 2000, 333; StV 2000, 382; OLG Karlsruhe NStZ-RR 2005, 223; OLG Hamm NStZ-RR 2010, 339; OLG Bamberg StV 2011, 421; dies passt nicht recht zusammen mit der gefestigten Rechtsprechung, wonach es im Interesse einer geordneten Strafrechtspflege liegen könne, dem - etwa bei großer Entfernung zwischen der Justizvollzugsanstalt des Verurteilten und dem Gericht - „unangemessenen Aufwand an Zeit und Arbeitskraft“ dadurch entgegenzuwirken, dass ein ersuchter Richter die mündliche Anhörung des Verurteilten durchführt, vgl. BGHSt. 28, 138, 141 ff.; OLG Düsseldorf NJW 1976, 256f.; Meyer-Goßner/Schmitt (Anm. 20), § 454 Rdn. 24; Graalmann-Scherer, in: Löwe/Rosenberg, StPO, 26. Aufl. 2010, § 454 Rdn. 33; die von einem Teil der Literatur und Rechtsprechung vertretene Gegenauffassung weist zu Recht auf den Zweck der mündlichen Anhörung hin, der bei Einschaltung eines ersuchten Richters regelmäßig verfehlt würde, vgl. OLG Koblenz MDR 1975, 863; OLG Rostock NStZ 2002, 109, 110; OLG Schleswig NJW 1975, 1131; Bringewat, Strafvollstreckung, 1993, § 454 Rdn. 42; Baier, in: Radtke/Hohmann (Anm. 55), § 454 Rdn. 18.

77 OLG Frankfurt a. M. NStZ-RR 2006, 357 f.; OLG Stuttgart NStZ-RR 2012, 323; vgl. schon Esser, NStZ 2003, 464 ff. Baier, in: Radtke/Hohmann (Anm. 55), § 454 Rdn. 20 will die Zulässigkeit einer Anhörung per Videokonferenz wohl nicht vom Einverständnis des Verurteilten abhängig machen. 78 OLG Frankfurt a. M. NStZ-RR 2006, 357 m.w. N. 
rung noch in Haft. Nähme man eine Pflicht der Vollstreckungsbehörde an, für die Dauer des Reststrafenaussetzungsverfahrens von einer Nachholung der Vollstreckung abzusehen, wären ausländische Gefangene mit Ausweisungsverfügung gleich zweifach im Vorteil gegenüber anderen Gefangenen: Sie würden im Verfahren nach $\S 456$ a StPO in der Regel früher als andere aus der Haft entlassen und könnten ihr Reststrafenaussetzungsverfahren im Bundesgebiet in Freiheit durchlaufen, ohne dass ein deutsches Gericht zuvor ihre Gefährlichkeit beurteilt hätte. Das würde sich mit dem gesetzgeberischen Willen nicht vertragen. Die Möglichkeit des Vollstreckungshaftbefehls soll dem Willen des Gesetzgebers zufolge den Verurteilten dauerhaft von einer Rückkehr nach Deutschland abhalten ${ }^{79}$. Wenn die Vollstreckungsbehörde also eine Aussetzung des Vollstreckungshaftbefehls ablehnt und der Verurteilte nach Deutschland einreisen möchte, ohne eine Inhaftierung zu befürchten, muss er abwarten, dass die einer Nachholung der Vollstreckung entgegenstehende Vollstreckungsverjährung nach § 79 StGB eintritt ${ }^{80}$.

\section{Fazit}

Die Rechtslage im Strafvollzug lässt in umfassenderem Maße eine Gleichbehandlung ausgewiesener Gefangener mit ihren Mitgefangenen zu, als sich dies in der aktuellen Vollzugspraxis - zumindest wie sie prima facie in den Verwaltungsvorschriften zum Strafvollzugsgesetz Widerhall findet und in der Literatur geschildert wird - widerspiegelt. Zur Umsetzung des verfassungsrechtlichen Resozialisierungsauftrags, der auch für ausgewiesene Gefangene streitet, kann die

79 BT-Drucks. 10/2720 S. 16.

80 Zur Unzulässigkeit der Vollstreckungsnachholung bei Vollstreckungsverjährung siehe MeyerGoßner/Schmitt (Anm. 20), § 456a Rdn. 6; Baier, in: Radtke/Hohmann (Anm. 55), § 456a Rdn. 7; Pfeiffer (Anm. 54), § 456a Rdn. 4; insoweit ist darauf hinzuweisen, dass das Vorgehen nach $\S 456$ a Abs. 1 StPO nach herrschender und zutreffender Auffassung keinen Vollstreckungsaufschub darstellt, der die Verjährung nach $\S 79$ a Nr. 2 lit. a StGB ruhen lässt, weil die Vollstreckungsaussetzung nicht als Aufschub gedacht ist: Eine gewisse Vorläufigkeit könnte man der Entscheidung nach § 456a Abs. 1 StPO allein aufgrund der Möglichkeit des Erlasses eines Vollstreckungshaftbefehls beimessen, jedoch handelt es sich nicht um einen Fall von § 79a Nr. 2 lit. a StGB, weil die Vollstreckungsbehörde die Entscheidung nach § 456a Abs. 1 StPO als endgültige trifft und mit dem Vollstreckungshaftbefehl nur den Verbleib des Verurteilten im Ausland absichern will. Ebenso Sternberg-Lieben/Bosch, in: Schönke/Schröder, StGB, 29. Aufl. 2014, § 79a Rdn. 4; Saliger, in: Nomos Kommentar, 4. Aufl. 2013, § 79a Rdn. 4; Lackner/Kühl, StGB, 28. Aufl. 2014, § 79a Rdn. 2; Fischer, StGB, 62. Aufl. 2015, § 79a Rdn. 5; Rosenau, in: Satzger/Schluckebier/ Widmaier, StGB, 2. Aufl. 2014, § 79a Rdn. 4; Jung, StV 2007, 106, 107; a. A. Schmid, in: Leipziger Kommentar, Band 3, 12. Aufl. 2008, § 79a Rdn. 4; Rudolphi/Wolter, in: Systematischer Kommentar zum StGB, § 79a Rdn. 2. 
Vollzugsanstalt auf das auf Wiedereingliederung in die deutsche Gesellschaft abzielende Behandlungsprogramm rekurrieren, zumal der Gefangene illegal in die Bundesrepublik zurückkehren oder im Ausland den deutschen Rechtskreis berührende Straftaten begehen könnte. Zudem dürften diese Wiedereingliederungsmaßnahmen sich regelmäßig auch dann als förderlich erweisen, wenn der Gefangene nach Haftverbüßung im Ausland lebt; ob auf Aggressionskontrolle abzielende Behandlungsmaßnahmen bei wegen Gewaltdelikten verurteilten Gefangenen wiedereingliederungsförderlich und im Einzelfall angezeigt sind, dürfte jedenfalls nicht davon abhängen, in welchem Land diese Gefangenen nach der Haft leben. Soweit ausgewiesene Gefangene gegenüber der Vollzugsanstalt nachvollziehbar darlegen, dass bei ihrer Wiedereingliederung landesspezifische Besonderheiten zu berücksichtigen sind, ist die Anstalt im Rahmen des ihr Zumutbaren $\mathrm{zu}$ entsprechender Berücksichtigung verpflichtet, wobei bei steigender Zahl Gefangener mit vergleichbarem Hintergrund innerhalb einer Anstalt auch das der Anstalt Zumutbare zunimmt. Außerdem verpflichtet das Resozialisierungsgebot zur Vermeidung von Depravationserscheinungen auch ausgewiesener Gefangener. Zwar besteht eine Vielzahl an Verwaltungsvorschriften, die ausgewiesene Gefangene gegenüber ihren Mitgefangenen schlechter stellen. Doch mit schlichtem Hinweis auf diese Verwaltungsvorschriften kann die Vollzugsanstalt die Versagung von Resozialisierungsmaßnahmen bei ausgewiesenen Gefangenen nur dann hinreichend begründen, wenn nach den Einzelfallumständen der gesetzliche Versagungsgrund offensichtlich erfüllt ist. In den anderen Fällen ist sie zu einer einzelfallbezogenen und substantiierten Entscheidungsbegründung verpflichtet.

In der Strafvollstreckung ist eine vom sonst üblichen Verfahrensgang abweichende Behandlung ausgewiesener Gefangener vom Gesetzgeber insofern vorgezeichnet, als er für diese Gefangenen das Verfahren nach § 456a StPO geregelt hat. Sieht die Vollstreckungsbehörde nach $\S 456$ a StPO bei einem ausgewiesenen Verurteilten von der weiteren Vollstreckung einer Freiheitsstrafe ab und erlässt zugleich einen Vollstreckungshaftbefehl für den Fall seiner Wiedereinreise in das Bundesgebiet, muss der Verurteilte bei einer Wiedereinreise auch dann mit seiner Inhaftierung rechnen, wenn er sich nunmehr aufenthaltsrechtlich legal im Bundesgebiet aufhält. Der Verurteilte muss auch dann mit einer Inhaftierung rechnen, wenn er nach Deutschland zwecks Durchführung eines Reststrafenaussetzungsverfahrens nach $\S 454$ StPO einreist. Die Vollstreckungsbehörde ist nicht verpflichtet, für die Dauer dieses Verfahrens den Vollstreckungshaftbefehl auszusetzen, weil der Verurteilte auch ohne eine solche Aussetzungsentscheidung am Reststrafenaussetzungsverfahren teilnehmen - insbesondere sein Recht auf rechtliches Gehör wahrnehmen - kann. Denn im Reststrafenaussetzungsverfahren befindet sich der Verurteilte typischerweise noch in Haft. Im Übrigen könnte, 
wenn man eine vollstreckungsbehördliche Aussetzungspflicht bejahte, ein Verurteilter sein Reststrafenaussetzungsverfahren im Bundesgebiet in Freiheit durchlaufen, ohne dass ein deutsches Gericht zuvor seine Gefährlichkeit beurteilt hätte. Eine Möglichkeit, dem Verurteilten die Einreise vor der gerichtlichen Entscheidung über die Reststrafenaussetzung zu ersparen, könnte darin liegen, im Ausland befindliche Verurteilte per Videokonferenz zu vernehmen, wenn die technischen Vorrichtungen vor Ort dies zulassen. Soweit nach aktueller Rechtsprechung der Fachgerichte die im Reststrafenaussetzungsverfahren gesetzlich vorgesehene mündliche Anhörung des Verurteilten grundsätzlich nicht per Videokonferenz durchgeführt werden könne, weil eine solche Konferenz dem Gericht nicht den erforderlichen persönlichen Eindruck vom Verurteilten vermitteln könne, erscheint dies angesichts technischer Fortschritte im Bereich der Telekommunikation immer fragwürdiger. 